\title{
Differentiated Vulvar Intraepithelial Neoplasia Associated with Intraepithelial Neoplastic Spread
}

\author{
Kien T Mai ${ }^{1 *}$ and Bojana Djordjevic ${ }^{2}$ \\ ${ }^{1}$ Department of Pathology and Laboratory Medicine, Canada
}

${ }^{2}$ Division of Anatomic Pathology, Sunnybrook Health Sciences Centre, Department of Pathobiology and Laboratory Medicine, Canada

*Corresponding author: KT Mai, Department of Pathology and Laboratory Medicine, Canada

\section{ARTICLE INFO}

Received: 陆 May 22, 2019

Published: May 29, 2019

Citation: Kien T Mai, Bojana Djordjevic. Association of Positive Results of Single Serum Biomarker with the Clinical Parameters. Biomed J Sci \& Tech Res 18(3)2019. BJSTR. MS.ID.003154.

Keywords: Vulvar; Intraepithelial; Neoplasia; Differentiated

Abbreviations: dVIN: Differentiated Vulvar Intraepithelial Neoplasia; VIN: Vulvar Intraepithelial Neoplasia; uVIN: Usual Vulvar Intraepithelial Neoplasia; SCC: Squamous Cell Carcinoma
ABSTRACT

Aims: Differentiated vulvar intraepithelial neoplasia (dVIN) associated with intraepithelial neoplastic spread and with usual vulvar intraepithelial neoplasia (UVIN) has not been studied.

Materials and Methods: Consecutive cases of vulvar intraepithelial neoplasia (VIN) diagnosed between 2011-2013 were reviewed. The neoplastic epithelia with or without accompanied invasive squamous cell carcinoma were categorized into: dVIN, UVIN and dVIN associated with scattered atypical neoplastic spread above the parabasal layer in the usual-like VIN pattern (u-like VIN). The lesions were examined with p16 immunostaining.

Results: Two- hundred-thirty-five consecutive cases of VIN including 54 invasive squamous cell carcinomas were identified. There were $12 \mathrm{dVIN}$ (invasive, $\mathrm{n}=8$, or $67 \%$ ), $178 \mathrm{uVIN}$ (invasive, $\mathrm{n}=5$ or $3 \%$ ) and $45 \mathrm{u}$-like VIN with or without uVIN (invasive, $\mathrm{n}=41$ or $91 \%)$. u-like VIN surface keratinisation exhibited features of keratinizing dysplasia. P16 was immunoreactive in $8 \%, 69 \%$ and $40 \%$ of dVIN, uVIN and u-like VIN lesions, respectively.

Conclusion: Like dVIN, u-like VIN is commonly associated with invasive squamous cell carcinoma. Therefore, its distinction from uVIN is of pathological and clinical significance.

\section{Introduction}

Squamous cell carcinoma (SCC) is the most common cancer of the vulva. It is believed that vulvar invasive SCC, similar to SCC of other body sites, is often preceded by premalignant vulvar intraepithelial lesions (VIN) [1-5]. These lesions are thought to evolve from abnormal basal keratinocytes which, with time, grow upwards to fill the epithelium, accounting for the well-known usual type of VIN (uVIN) . Eventually they acquire the ability to break through the basement membrane and invade into the stroma. This pathway has been best studied in the cervix, but it has also been applied to other squamous /epithelial sites including the bronchus, esophagus and oral cavity [6-8]. uVIN is most often associated with oncogenic HPV. Another distinct pathway towards invasive SCC, found in differentiated vulvar intraepithelial neoplasia (dVIN), involves cytological atypia limited to the basal and parabasal cells. This pathway has been described in the anal canal and anterior oral cavity [9-11]. dVIN lesions are more frequently associated with invasive SCC than UVIN, are often p53 positive by immunohistochemistry, and should be thought of as high grade, despite their subtle histological features [12-14]. dVIN is the type most often associated with untreated or poorly controlled vulvar lichen sclerosus. Since dVIN developed from neoplastic transformation of basal/parabasal cells with early stromal malignant invasion, we hypothesized that there may exist a varying level of upward and intraepithelial spread of dysplastic squamous cells that may mimic uVIN. This study aimed to investigate whether or not the vulvar SCC harbours the above intraepithelial spread, and if present, its clinical and pathological significance. An Immunohistochemical panel consisting of p16, and p53 was also employed as these markers have been shown to distinguish between the differentiated and classic pathways at other sites. 


\section{Materials and Methods}

Ethics approval from the investigational review board of our medical center was obtained. The database at the Department of Anatomical Pathology at the Ottawa Hospital was searched for SCC and vulvar intraepithelial neoplasia diagnosed at our institution between 2011-2013. The patients' ages were obtained from the pathology reports. All tissues had been routinely fixed in $10 \%$ buffered formalin and embedded in paraffin. All available hematoxylin-eosin slides were reviewed with particular attention to the surface epithelium. Characterization of intraepithelial neoplastic lesions was done according to the Lower Anogenital Squamous Terminology (LAST) [7]. In addition, three types of VIN were defined as follows (Figure $1 \mathrm{~A}-\mathrm{C}$ ):

a) dVIN characterized by keratinocyte atypia limited to the basal and parabasal cells. dVIN is characterized by the lack of upward extension of atypia, however a typia does frequently extend into rete pegs. This type of neoplastic epithelium is frequently (Figure 1A).

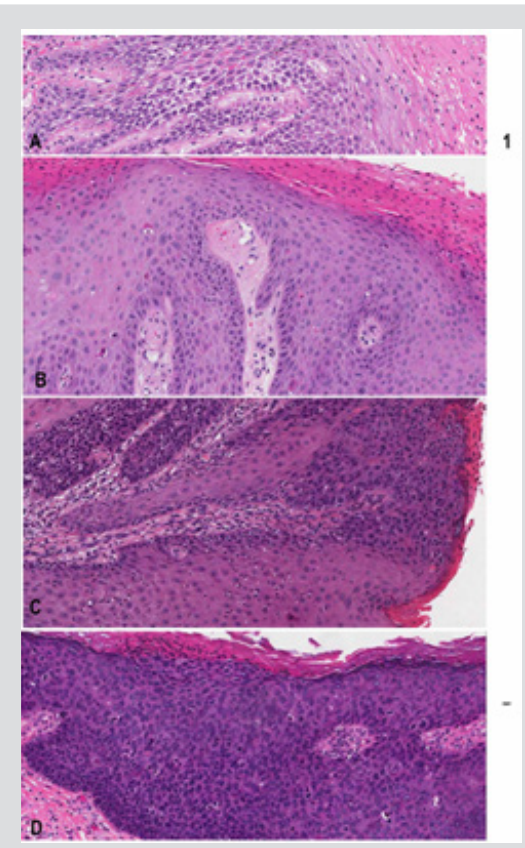

Figure 1: Representative images for comparative study of different vulvar intraepithelial neoplasia

a) dVIN displaying atypical cells limited in the basal/ parabasal layer with the remarkable intercellular widening and \the unremarkable mid and upper epidermis b) dVIN with diffuse intraepithelial spread (or u-like VIN). Note in the left third of the micrograph showing dVIN with atypical cells in the basal/parabasal layer and in the left right two-third showing dVIN with presence of atypical cells in the upper epidermis mimicking high grade uVIN associated with parakeratosis imparting the term of keratinizing dysplasia

c) dVIN with focal/patchy intraepithelial spread (u-like VIN). Note dVIN features in the basal/parabasal layer

d) uVIN showing the neoplastic involvement full thickness of the epithelium. b) dVIN with upward (and intraepithelial) neoplastic cell spread in b) diffuse (Figure 1B) or c) focal (Figure 1C) manner mimicking uVIN or "usual-like VIN" (u-like VIN) [15].

c) high grade usual VIN (uVIN) has nuclear pleomorphism and hyperchromasia of the keratinocytes that extends upwards to involve the superficial half of the epithelium, (Figure 1D). High grade uVIN corresponds to VIN2 /3 is commonly associated with a high-risk HPV-infection.

Other features helpful in the characterization of dVIN and highgrade u-like VIN are:

a) Cellular atypia, commonly mild to moderate

b) Early keratinization and

c) Intercellular space widening, creating the "cobble stone appearance", and

d) Fewer atypical cells in the upper part of the epithelium in comparison to uVIN.

Immunohistochemical studies were performed on representative sections with mucosa overlying or adjacent to invasive SCC. Immunohistochemistry was carried out with a BOND-MAX automated system (Leica MicroSystems, Wetzlar, Germany) for p16 (dilution 1:500, catalogue SC-56330, p53 (dilution: ready to use, catalogue: PA0057, Leica). Tissue sections were subject to epitope retrieval using the Bond epitope retrieval solution (Leica) appropriate to the primary antibody. Primary antibody binding was detected using the Bond Polymer Refine Detection system (Leica) which is biotin-free. This system exposes tissue sections to primary antibody for 15 minutes, post-primary solution for 8 minutes, Bond polymer for 8 minutes, peroxide block for 5 minutes, 3-diaminobenzidine (DAB) chromogen for 10 minutes and hematoxylin counter stain for 7 minutes. A positive control was included on each slide. p16 immunostaining was scored as positive when the epithelium demonstrated strong nuclear or cytoplasmic staining involving diffuse and full or nearly full thickness of the epithelium. Immunoreactivity for p53 required a e $3+$ nuclear staining to be considered positive. For p53, two patterns of positive immunoreactivity recorded : a) Linear p53 linear immunostaining characterized by strong nuclear staining of at least $25 \%$ of the observed cells and b) scattered type characterized by weaker and diffuse immunoreactivy in the observed area of the epithelium [16]. Of note, p53 immunostaining may be related to ischemic stress in lichen sclerosus [17] and reactive changes. Statistical analysis was performed by using Sisa software. The $p$-value $<0.05$ was considered as statistically significant

\section{Results}

A total of 235 patients with VIN including 54 invasive SCC of the external genitalia were identified. The mean patient age at the time of diagnosis was $58 \pm 19$ years (range 22-93). Table 1 summarizes the clinical and pathological characteristics of all cases included in the final analysis. All of these cases demonstrated evidence of 
intraepithelial neoplasia, either dVIN, uVIN or a mixture of dVIN/ulike VIN and UVIN . Specifically, changes consistent with pure dVIN were present in 12 cases ( 8 with invasive SCC); changes consistent with uVIN were present in 178 cases (5 with invasive SCC) while; 45 cases demonstrated features of both dVIN/u-like VIN and uVIN
(41 with invasive SCC). Of 51 cases of invasive SCC, a history of lichen sclerosus was recorded in 8 out of 40 patients with available clinical and pathological information. There were 36 patients with a history of cervical intraepithelial neoplasia or HPV of the anal canal.

Table 1: Summary of Clinical and Pathological Findings.

\begin{tabular}{|c|c|c|c|c|c|c|c|c|}
\hline & \multirow{2}{*}{ No } & \multirow{2}{*}{ Age } & \multicolumn{4}{|c|}{ pT stage } & \multirow{2}{*}{ Lymph node } & \multirow{2}{*}{ p16\% } \\
\hline & & & is & 1a & $1 \mathrm{~b}$ & 2 & & \\
\hline DSIN & $12(5 \%)$ & $77 \pm 14(53-93)$ & 4 & 2 & 6 & & 0 & $8 \%$ \\
\hline Mixed* & 45 (19\%) & $68 \pm 12(47-90)$ & 3 & 9 & 30 & 2 & 2 & (23)51\% \\
\hline u-like VIN & 10 & $72 \pm 11(59-88)$ & 1 & 1 & 7 & 1 & & (6) $60 \%$ \\
\hline dVIN+uVIN & 17 & $63 \pm 15(47-90)$ & & 2 & 14 & 1 & & (11) $65 \%$ \\
\hline Mostly dVIN** & 18 & $69 \pm 9(53-84)$ & 2 & 6 & 9 & & & $2(4) \%$ \\
\hline USIN & $178(76 \%)$ & $57 \pm 16(22-75)$ & 173 & 1 & 2 & 2 & 1 & $69 \% * * *$ \\
\hline Total & 235 & $58 \pm 19(22-93)$ & 180 & 12 & 38 & 4 & 3 & \\
\hline
\end{tabular}

Note:

${ }^{*}$ Mixed with u-like VIN: uVIN accounting less than $10 \%$

Mixed with dVIN+ VIN: dVIN/u-like VIN or uVIN accounting from 10-90\%

Mixed with mostly uVIN: uVIN accounting less than $10 \%$

**dVIN accounting for more than $90 \%$ of neoplastic epithelium.

***Rate based only 49 cases with available p16 Immunostaining.

Group 1: Clinical and Pathological Features of Cases with Differentiated Vulvar Intraepithelial Neoplasia with or without Invasive SCC

Changes consistent with dVIN were found in 12 cases (5\%). The cases were further divided into the following $\mathrm{T}$ stage groups: pTis (no invasive SCC): 4, pT1a: 2, pT1b:6 and pT2:0. Lymph node metastases were absent. The mean age was $77 \pm 14$ years (range: 53 - 93). In these cases, the surface epithelium was flat in 10 cases, while it was polypoid or condylomatous/papillary in 2 cases. The epithelium frequently demonstrated mild to moderate and occasionally marked cytologic atypia limited to the basal/parabasal layers, large eosinophilic cytoplasm, pronounced intracellular spaces and early keratosis (imparting a 'cobblestone' appearance). Despite the fact that atypical cells remained in the lower part of the epithelium, disordered nuclear arrangement was evident. The involved squamous epithelium showed atrophic change with frequent ulceration (Figure 1A). The rete ridges were identified in a majority of cases which were often angulated, with a "clubshape" appearance and were irregular in size and in architectural distribution. Discernible intercellular widening was often extensive and was focal in one case.

Group 2: Histological Features of Cases with Mixed Differentiated/Usual-Like and Usual Vulvar Intraepithelial Neoplasia

Changes consistent with mixed dVIN and u-like VIN were found in 45 cases (19\%). The cases were further divided into the following T stage groups: pTis: 4, T1a: 9, pT1b: 30, pT2: 2: Lymph node metastases were found in 2 cases. The mean age was

$68 \pm 12$ years (range: $47-90$ ). The difference between rates of development of invasive SCC for mixed type and usual type of VIN was statistically significant $(p<0.01)$. In this group 3 , the surface epithelia showed varying proportion of an admixture of dVIN and u-like VIN. Specifically. Predominantly dVIN and u-like VIN (with or without focal uVIN in less than $10 \%$ of neoplastic epithelium): 10 cases (Figure 2). Significant areas of dVIN /u-like VIN or uVIN each accounting from 10-90\% of neoplastic epithelium : 17 cases (Figure 3). Predominantly uVIN (with focal dVIN/u-like VIN less than $10 \%$ of neoplastic epithelium): 18 cases.

Group 3: Clinical and Pathological Features of Cases with Usual (typical) Vulvar Intraepithelial Neoplasia (uVIN)

Changes consistent with uVIN were found in 178 cases (76\%). The cases were further divided into the following $\mathrm{T}$ stage groups: pTis: 173, pT1a: 1, pT1b:2, pT2:2. Lymph node metastases were found in 1 case. The mean age was $57 \pm 16$ years (range: $22-75$ ). In these cases, the overlying epithelium demonstrated full thickness changes with disordered nuclear arrangement and severe cytologic atypia. Intercellular widening was not discernible but focal koilocytosis may be demonstrated (Figure 1D). In comparison to the adjacent surface dVIN, the u-like VIN epithelium showed: a) intercellular widening with cobble stone appearance in the basal/parabasal layer as in dVIN b) occasional irregular papillary surface architecture (whereas dVIN epithelium was flat), c) frequent keratinization of the uppermost third imparting for the term "keratinizing type of high grade squamous dysplasia" (Figures 2C \& 3C). In addition, u-like VIN epithelium transformed or merged into uVIN epithelium 
in 17 cases with coexisting uVIN (Figure 3A). Note that invasive SCC was not only connected the with VIN involving the full or nearly full thickness of epithelium but also with VIN limited to the lower half of the epithelium and dVIN (Figures 2-4).

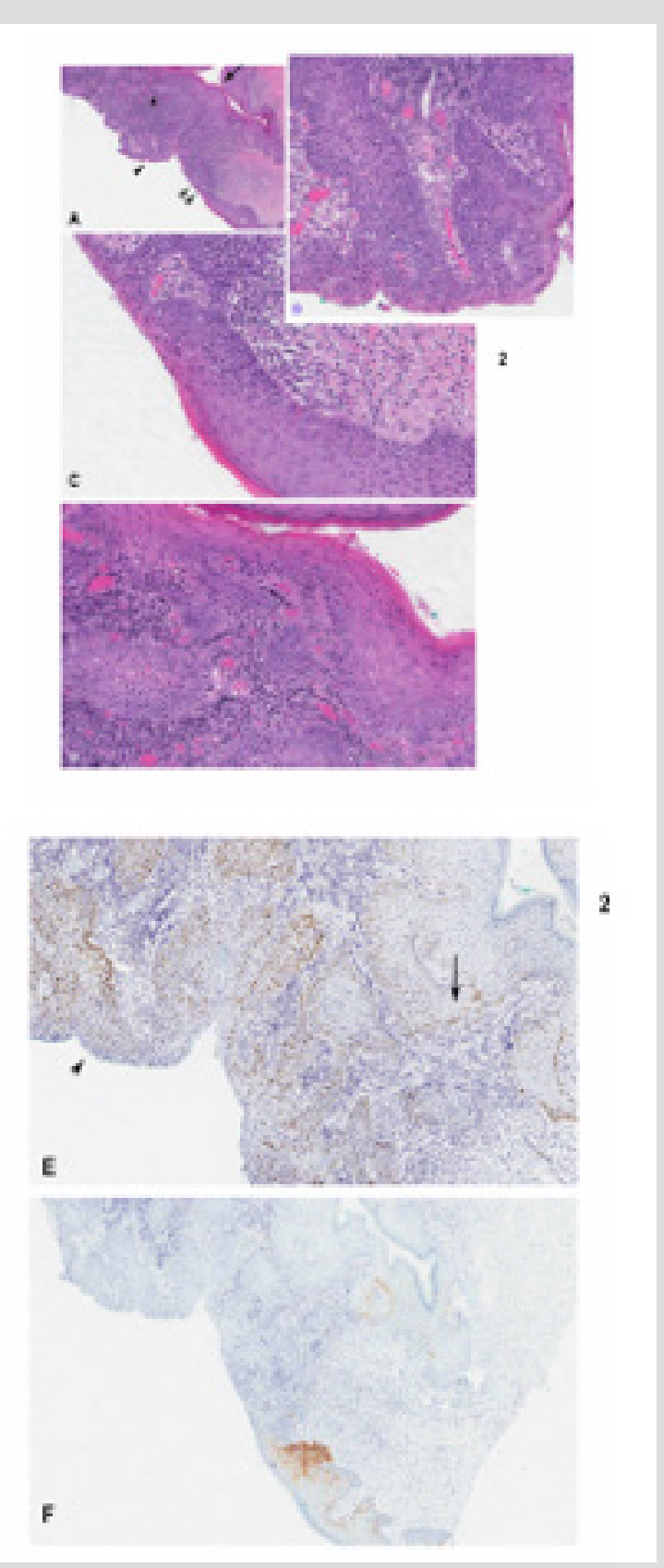

Figure 2: A case of nonreactive-p16 intraepithelial neoplasia displaying dVIN with intraepithelial spread and invasive carcinoma in a case of negative p16 immunoreactivity

a) Low magnification showing invasive carcinoma (asterix) developing from the neoplastic overlying epithelium

b) High magnification of area in A indicated by arrowhead double arrowhead

c) Single arrowhead

d) Showing u-like VIN dVIN with gradual change into u-like VIN and dVIN. Note invasive carcinoma is connected with VIN in B,C and D.

e) Positive p53 immunoreactivity limited to basal/parabasal layer in dVIN (arrow) and more diffuse in u-like VIN (arrowhead)

f) Negative p16 immunoreactivity. 


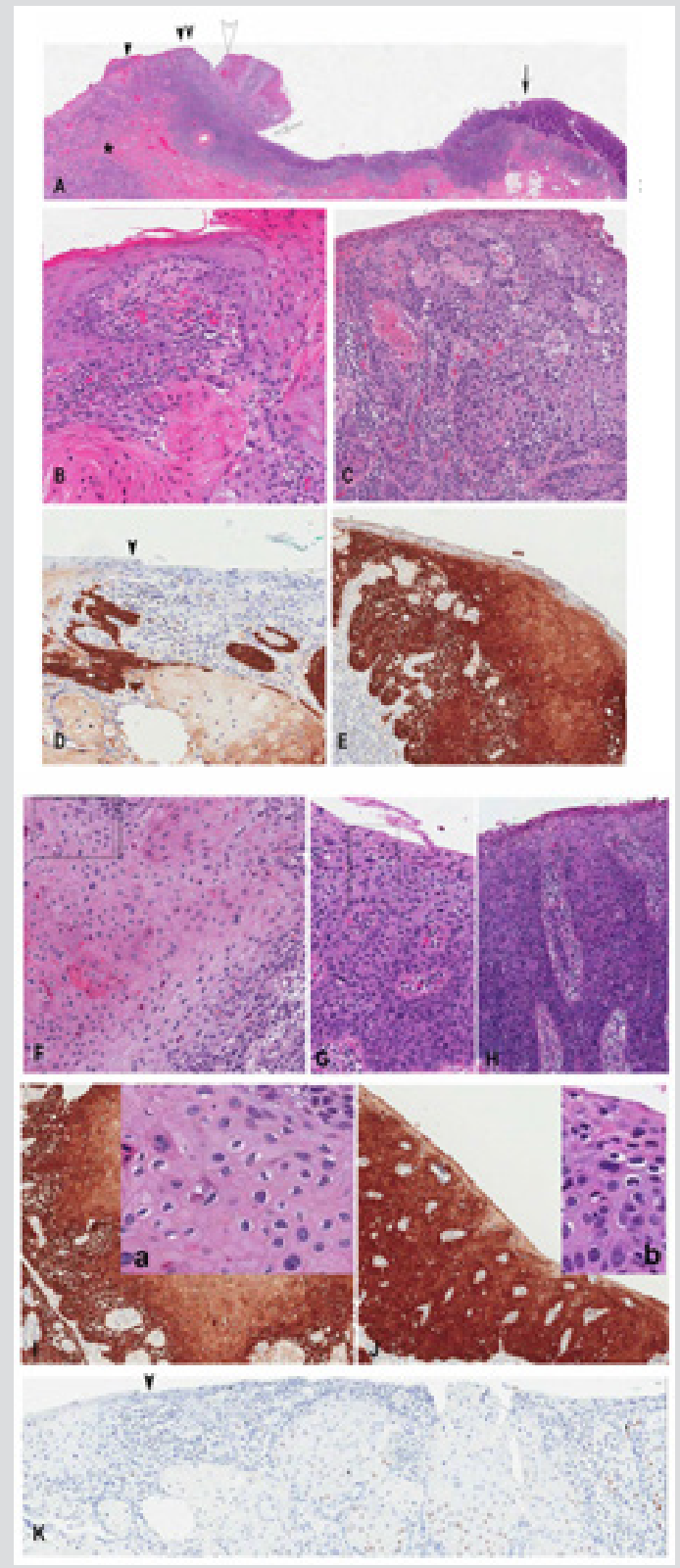

\section{Figure 3:}

a) $3 \mathrm{~cm}$ vulvar lesion with T1b invasive SCC developing from the dVIN on the left side and intraepithelial spread (u-like VIN) (arrowheads) and uVIN on the right side (solid arrow). Note the condylomatous formation (open arrowhead) and koilocytosis (insets a and b) associated with u-like VIN

b) A high magnification of the area indicated by black arrowhead in A) showing dVIN.

c) A high magnification of the area indicated by double black arrowhead in A showing u-like VIN with the attached invasive SCC (asterix in A). Note the absence of koilocytosis.

d) Immunostaining for p16 of the area corresponding to B showing focal but positive p16 reactivity

e) area corresponding to $\mathrm{C}$ showing positive $\mathrm{p} 16$ reactivity

f) A high magnification of the area indicated by open arrowhead in A) showing u-like VIN with condylomatous changes and koilocytosis,

g) A high magnification of the area indicated by solid arrow in A) showing high grade uVIN koilocytosis and (H) a high magnification of the area indicated by open arrow in A) showing in situ carcinoma (without koilocytosis). Note the focal and barely discernible intercellular widening the absence of invasive SCC in the underlying stroma

h) Immunostaining for p16 of the area corresponding to $F$ and $G$ showing positive p16 reactivity .

i) Insets a and $\mathrm{b}$ showing koilocytosis in areas delineated by rectangles in $\mathrm{F}$ and $\mathrm{G}$

j) Immunostaining for p53 showing negative reactivity. 


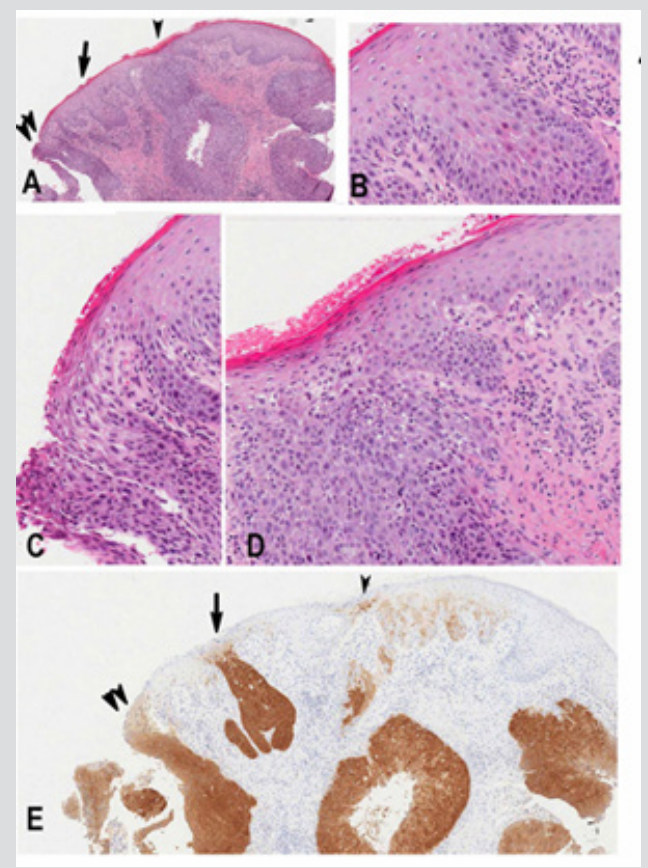

Figure 4: A case of p16-reactive invasive carcinoma developing from overlying p16- nonreactive dVIN with focal intraepithelial spread. the neoplastic lesion was p53-nonreactive (not shown)

a) Low magnification

b) High magnifications of areas of (B) and (C) indicated by arrow and arrowhead in A and E showing dVIN, (D) indicated by double arrowhead in A and E showing focal intraepithelial spread.

c) P16 immunostaining showing reactivity in invasive carcinoma and focal intraepithelial spread only.

\section{Immunohistochemistry}

p16 was reactive in $1 / 12(8 \%)$ cases of typical dVIN (Gand in $34 / 49(69 \%)$ cases of uVIN . For the Group 2, p16 was reactive in 18/45 (51\%) cases (Figure 3DEIJ) and non-reactive in the remaining cases (Figure 2F). In cases with p16-reactive surface epithelium, the immunoreactivity was observed in surface epithelia of uVIN and u-like VIN and only occasionally dVIN epithelia displayed focal positive p16 immunostaining (Figure 4E). In addition, in these cases with p16-reactive surface epithelium, the underlying invasive SCC was p16-reactive (Figure 4E). Immunostaining for p53 for Group 1, demonstrated linear reactivity in the basal and parabasal layers in dVIN. In u-like VIN and UVIN, reactivity for p53 was often more diffuse in p16 non-reactive cases (Figure 2E) and negative or diffuse but diminished in epithelia with positive p16 reactivity (Figure 3K).

\section{Discussion}

In this study, invasive SCC was observed to develop from the overlying squamous epithelium displaying three types of VIN: dVIN, uVIN and mixed dVIN/u-like VIN+uVIN; which accounted for $5 \%, 76 \%$ and $19 \%$ of the cases respectively. In uVIN, the neoplastic cells expand from the basal layer to involve the full thickness of the epithelium. uVIN is known to represent the most common pathway of HPV-driven carcinogenesis as evidenced by the positive immunoreactivity for $\mathrm{p} 16$, a surrogate marker of high risk HPV infection $[16,18]$. This type of uVIN also includes the basaloid histological pattern of VIN, also described as a variant of simplex ("differentiated") VIN. The basaloid VIN is characterized by a homogeneous population of small, "undifferentiated" keratinocytes, which involve the full thickness of the epithelium and are diffusely positive for p16INK4a and negative for p53 [19]. Vulvar dVIN has been shown to be morphologically and immunohistochemically similar to the analogous lesions in the oropharynx and in actinic keratosis [20,21]. At molecular level, mutations of p53 are frequently demonstrated. The atypical nuclei of dVIN were more frequently limited to the basal/parabasal layer suggesting that the neoplastic process develops primarily from the basal /parabasal layers with limited or absent tendency for expansion into the upper layers of the epidermis. As a result, dVIN commonly known for the high potential of stromal invasion [14]. In addition, in dVIN epithelium, intercellular widening was limited almost only to the neoplastic areas (basal/parabasal layers) in band/ribbon-like in the squamous epithelium. The intercellular widening is likely related to extracellular edema associated neoplastic cells of dVIN or u-like VIN. Although the above change is not specific but is characteristic and is not commonly seen in typical uVIN.

Although the largest proportion of VIN was uVIN, the largest proportion of vulvar invasive SCC was observed to develop from overlying surface epithelia showing dVIN and an admixture of dVIN and u-like VIN. dVIN is widely believed to develop from lichen sclerosus $[22,23]$ and UVIN is frequently driven by high-risk HPV 
and dVIN [24,25], dVIN has been demonstrated occasionally to be reactive for $\mathrm{p} 16$ [2]. $\mathrm{u}$-like VIN can be associated with $\mathrm{p} 16$ reactivity as evidenced in the present study. Therefore, in the two pathways of development of vulvar intraepithelial neoplasia in the external genitalia, as in the oral cavity:

A. uVIN has a low potential for development of invasive SCC (occurring late in the course of the disease). The neoplastic parabasal/basal cells retain the potential of orientation with upward progression to the superficial cell layers.

B. dVIN has an early potential for development invasive SCC. The neoplastic parabasal/basal cells lose the potential of orientation in the upward/orderly progression toward the superficial layer of the epithelium and ortho-keratosis . As a result, these neoplastic cells show:

a) Propensity of inverted (downward) growth and frequent early keratosis as seen in pure dVIN, or

b) Propensity of inverted (downward) growth and disorderly intraepithelial and upward spread mimicking uVIN (u-like VIN) and frequent keratosis (keratinizing dysplasia). The term "keratinizing dysplasia" has been used in the head and neck pathology to designate premalignant lesion associated with invasive carcinoma but not displaying full thickness epithelial involvement $[26,27]$. This term was also used in the lower genital tract [28-30].

c) A combined pathway with an independent or secondary development of dVIN in the background of uVIN as evidenced by small areas of dVIN/u-like VIN within large areas of uVIN. U-like VIN associated with p16-reactive uVIN also exhibit p16 immunoreactivity as seen Figures 3D \& 3J.

In u-like VIN, the neoplastic cells in the upper layer of the epithelium (displaying dVIN features) likely represent the secondary spread of neoplastic cells of dVIN. The possible focal retrograde invasion of SCC into the overlying epithelium cannot be completely excluded, however this phenomenon is not evident in most SCC associated with uVIN. As a result, the stromal invasion is independent from the spreading of neoplastic cells in the epithelium. This phenomenon of intraepithelial spread is well known in melanoma of superficial spread type (in contrast to lentigo maligna melanoma with malignant cells limited in the basal layer but associated with invasion into the stroma). Furthermore, in the urinary bladder, in situ and invasive urothelial carcinomas represent two separate pathways of neoplastic transformation from neoplastic urothelial basal cells [31]. Similar to ductal spread of prostatic carcinoma [32], vulvar intraepithelial malignant spread is almost always associated with invasive SCC and often at advanced stage of disease. HPV infection has been demonstrated to alter p53 activity. HPV proteins E6 form a complex with AP proteins that targets p53 for complete or incomplete degradations via the ubiquitin dependent proteolytic system [33-35]. Since p53 is considered as "gatekeeper
" or "caretaker" of the cell proliferation, the mutation of p53 (seen in non-HPV infected dVIN) or the degradation with a complete or incomplete loss of p53 activity (caused by HPV in uVIN) play equal role in the carcinogenesis of dVIN. Therefore, the mixed neoplastic epithelia of dVIN and HPV-driven uVIN and the p16-reactive invasive carcinoma are the evidence of the role of HPV infection in the histopathogenesis of dVIN.

Although, molecular investigation was not performed in this study, the typical condylomatous and koilocytotic changes in the epithelia (associated with dVIN) as shown in the Figure 3 are almost diagnostic of HPV infection. This proposed mechanism is supported by the reports of dVIN showing p16 reactivity or dVIN associated with negative p53 reactivity $[25,30]$. In conclusion, the histopathological and immunohistopathological studies suggested that dVIN plays a major role in the histopathogenesis of invasive vulvar SCC. u-like VIN represents keratinizing squamous dysplasia and the advanced dVIN that is most often associated with invasive SCC. The thickness/level of the atypical cells above the parabasal layer in u-like VIN merely represent an epiphenomenon or a surrogate marker of dVIN at advanced stage with invasive carcinoma. Distinction of uVIN from u-like VIN and admixture of u-like VIN and dVIN is of clinical significance due to the low incidence of invasive SCC in pure uVIN. Molecular studies with HPV typing are necessary for further investigation of the significance of u-like VIN and keratinizing squamous dysplasia since P16 protein is also up-regulated in conditions other than high risk HPV infection.

\section{References}

1. Reyes MC, Cooper K (2014) An update on vulvar intraepithelial neoplasia: terminology and a practical approach to diagnosis. J Clin Pathol 67(4): 290-294.

2. Dong F, Kojiro S, Borger DR, Growdon WB, Oliva E (2015) Squamous Cell Carcinoma of the Vulva: A Subclassification of 97 Cases by Clinicopathologic, Immunohistochemical, and Molecular Features (p16, p53, and EGFR). Am J Surg Pathol 39(8): 1045-1053.

3. Trietsch MD, Nooij LS, Gaarenstroom KN, van Poelgeest MI (2015) Genetic and epigenetic changes in vulvar squamous cell carcinoma and its precursor lesions: a review of the current literature. Gynecol Oncol 136(1): 143-157.

4. Preti M, Scurry J, Marchitelli CE, Micheletti L (2014) Vulvar intraepithelial neoplasia. Best Pract Res Clin Obstet Gynaecol 28(7): 1051-1062.

5. Van Esch EM, Dam MC, Osse ME, van Esch EM, Dam MC, et al. (2013) Clinical characteristics associated with development of recurrence and progression in usual-type vulvar intraepithelial neoplasia. Int J Gynecol Cancer 23(8): 1476-1483

6. Crissman JD, Zarbo RJ (1989) Dysplasia, in situ carcinoma, and progression to invasive squamous cell carcinoma of the upper aerodigestive tract. Am J Surg Pathol 13(Suppl 1): 5-16.

7. Darragh TM, Colgan TJ, Thomas Cox J Heller DS, Henry MR, Luff RD et al. (2013) The Lower Anogenital Squamous Terminology Standardization project for HPV-associated lesions: background and consensus recommendations from the College of American Pathologists and the American Society for Colposcopy and Cervical Pathology. Int J Gynecol Pathol 32(10): 76-115.

8. van Hulst AM, Kroon W, van der Linden ES (2015) Grade of dysplasia and malignant transformation in adults with premalignant laryngeal lesions. Head Neck 38(suppl 1): E2284-2290. 
9. Paliga A, Mai KT (2014) Squamous cell carcinomas of the anterior oral cavity are commonly associated with simplex (or differentiated) oral intraepithelial neoplasia: clinical and pathologic significance. Int J Surg Pathol 22(3): 231-240.

10. Wasserman JK, Bateman J, Mai KT (2016) Differentiated Squamous Intraepithelial Neoplasia Associated with Squamous Cell Carcinoma of The Anal Canal. Histopathology 68(6): 834-842.

11. Arsenic R , Kurrer MO (2013) Differentiated dysplasia is a frequent precursor or associated lesion in invasive squamous cell carcinoma of the oral cavity and pharynx. Virchows Arch 462(6): 609-617.

12. Mulvany NJ, Allen DG (2008) Differentiated intraepithelial neoplasia of the vulva. Int J Gynecol Pathol 27(1): 125-135.

13. Ruhul Quddus M, Xu C, Steinhoff MM, Zhang C, Lawrence WD, et al. (2005) Simplex (differentiated) type vin: Absence of p16ink4 supports its weak association with hpv and its probable precursor role in non-hpv related vulvar squamous cancers. Histopathology 46(6): 718-720.

14. Eva LJ, Ganesan R, Chan KK, Honest H, Luesley DM (2009) Differentiated type vulval intraepithelial neoplasia has a high-risk association with vulval squamous cell carcinoma. J Gynecol Cancer 19(4): 741-744.

15. Mai KT, Hogan K, Wasserman JK (2016) Laryngeal Squamous Cell Carcinomas are commonly associated with differentiated squamous intraepithelial neoplasia with or without an admixture with usual type of squamous intraepithelial neoplasia: clinical and pathologic significance. Appl Immunohistochem Mol Morphol 26(5): 351-359.

16. Klaes R, Benner A, Friedrich T (2002) P16INK4a immunohistochemistry improves interobserver agreement in the diagnosis of cervical intraepithelial neoplasia. The Am J Surg Pathol 26(11): 1389-1399.

17. Liegl B, Regauer S (2006) p53 immunostaining in lichen sclerosus is related to ischaemic stress and is not a marker of differentiated vulvar intraepithelial neoplasia (d-VIN). Histopathology 48(3): 268-274.

18. Gültekin SE, Sengüven B, Klussmann JP, Dienes HP (2015) P16(INK 4a) and Ki-67 expression in human papilloma virus-related head and neck mucosal lesions. Invest Clin 56(1): 47-59.

19. Ordi J, Alejo M, Fusté V, Lloveras B, Del Pino M, et al. (2009) HPV-negative vulvar intraepithelial neoplasia (VIN) with basaloid histologic pattern: an unrecognized variant of simplex (differentiated) VIN. Am J Surg Pathol 33(11): 165916-165965.

20. Bagazgoitia L, Cuevas J, Juarranz A (2010) Expression of p53 and p16 in actinic keratosis, bowenoid actinic keratosis and Bowen's disease. J Eur Acad Dermatol Venereol 24(2): 228-230.

21. Purgina B, Hassan M, Olberg B, Lai CK, Mai KT (2015) Histopathogenesis of non-HPV-related differentiated oral squamous intraepithelial neoplasia. Appl Immunohistochem Mol Morphol 23(4): 273-279.

22. van den Einden LC, de Hullu JA, Massuger LF (2014) These comprise a more common HPV-related usual type VIN (also referred to as classic, undifferentiated, basaloid, warty, Bowenoid type) and a more

\section{ISSN: 2574-1241}

DOI: 10.26717/BJSTR.2019.18.003154

KT Mai. Biomed J Sci \& Tech Res

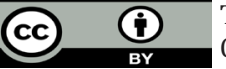

This work is licensed under Creative

Commons Attribution 4.0 License

Submission Link: https://biomedres.us/submit-manuscript.php uncommon differentiated (simplex) type which is non-HPV related and which is sometimes associated with lichen sclerosus J Clin Pathol 67: 290-294.

23. van de Nieuwenhof HP, Johan Bulten J, Hollema H (2011) Differentiated vulvar intraepithelial neoplasia is often found in lesions, previously diagnosed as lichen sclerosus, which have progressed to vulvar squamous cell carcinoma. Modern Pathol 24(2): 297-305.

24. Cheng AS, Karnezis AN, Jordan S, Singh N, McAlpine JN, et al. (2015) p16 Immunostaining Allows for Accurate Subclassification of Vulvar Squamous Cell Carcinoma Into HPV-Associated and HPV-Independent Cases. 16 as HPV marker. Int J Gynecol Pathol 35(4): 385-393.

25. Dong F, Kojiro S, Borger DR, Growdon WB, Oliva E (2015) Squamous Cell Carcinoma of the Vulva: A Subclassification of 97 Cases by Clinicopathologic, Immunohistochemical, and Molecular Features (p16, p53, and EGFR). Am J Surg Pathol 39(8):1045-1053.

26. Wenig BM (2017) Squamous cell carcinoma of the upper aerodigestive tract: dysplasia and select variants. Mod Pathol 30(s1): S112-S118.

27. Wenig BM (2002) Squamous cell carcinoma of the upper aerodigestive tract: precursors and problematic variants. Mod Pathol 15(3): 229-254.

28. De Vuyst H, Clifford GM, Nascimento MC, Madeleine MM, Franceschi S (2009) Prevalence and type distribution of human papillomavirus in carcinoma and intraepithelial neoplasia of the vulva, vagina and anus: a meta-analysis. Int J Cancer 124(7): 1626-1636.

29. Boggs EW, Burgis JT, Spiryda LB (2012) Keratinizing squamous dysplasia pap test: a case for colposcopy. J Low Genit Tract Dis 16(1): 30-33.

30. Singh N, Leen SL, Han G, Faruqi A, Kokka F, et al. (2015) Expanding the morphologic spectrum of differentiated VIN (dVIN) through detailed mapping of cases with p53 loss. Am J Surg Pathol 39(1): 52-60.

31. Blanes A, Rubio J, Sanchez Carrillo JJ Diaz-Cano SJ (2009) Coexistent intraurothelial carcinoma and muscle-invasive urothelial carcinoma of the bladder: clonality and somatic down-regulation of DNA mismatch repair. Hum Pathol 40(7): 988-997.

32. Chen Z, Chen N, Shen P, Gong J, Li X, et al. (2015) The presence and clinical implication of intraductal carcinoma of prostate in metastatic castration resistant prostate cancer. Prostate 75(12):1247-1254.

33. Nuber U, Schwarz SE, Scheffner M (1998) The ubiquitin-protein ligase E6-associated protein (E6-AP) serves as its own substrate. Eur J Biochem 254(3): 643-649.

34. Helland A, Karlsen F, Due EU, Holm R, Kristensen G, et al. (1998) Mutations in the TP53 gene and protein expression of p53, MDM 2 and $\mathrm{p} 21 /$ WAF-1 in primary cervical carcinomas with no or low human papillomavirus load. Br J Cancer 78(1): 69-72.

35. Brimer N, Vande Pol SB (2014) Papillomavirus E6 PDZ interactions can be replaced by repression of p53 to promote episomal human papillomavirus genome maintenance. J Virol 88(5): 3027-3030.

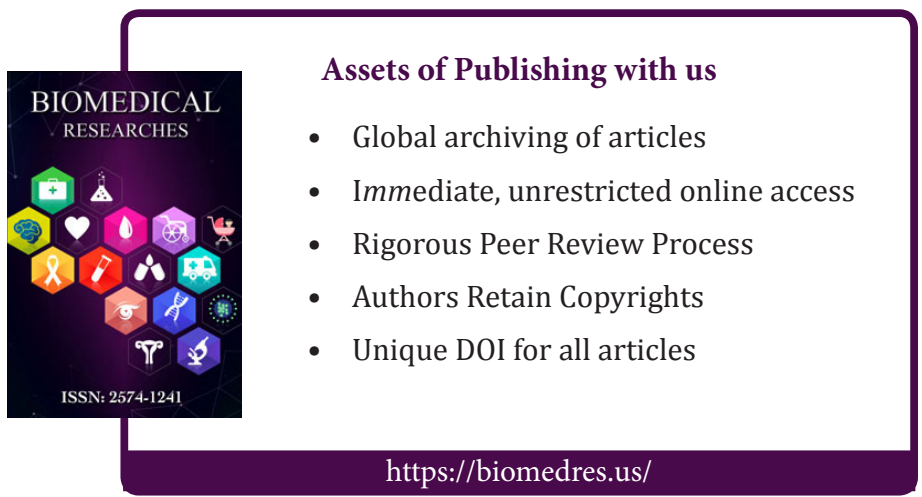

